\title{
Learning style preferences of medical students: a single-institute experience from Saudi Arabia
}

\author{
Ayesha Nuzhat, Raneem 0. Salem, Mohammed S.A. Quadri, Nasir Al-Hamdan
}

Department of Basic Medical Sciences, Faculty of Medicine, King Fahad Medical City, King Saud Bin Abdul Aziz University for Health Sciences, Kingdom of Saudi Arabia

Correspondence: Ayesha Nuzhat, Faculty of Medicine, King Fahad Medical City, King Saud Bin Abdul Aziz University for Health Sciences. Email: anuzhat11@gmail.com

Accepted: August 01, 2011

\begin{abstract}
Objectives: The purpose of this study was to examine preferred learning styles of undergraduate medical students at King Saud Bin Abdul Aziz University for Health Sciences, King Fahad Medical College, Saudi Arabia.

Methods: A cross sectional study of preclinical students with 74 male and 72 female $(n=146)$ was performed. The validated VARK questionnaire was used to categorize the learning styles of students. The questionnaire consists of 16 items which identify four different learning styles: visual, aural, reading/writing and kinesthetic. Descriptive statistics were used to identify the learning styles of students.
\end{abstract}

Results: The response rate was $70 \%$. The results showed that $5.5 \%, 11.6 \%, 2.1 \%$, and $8.2 \%$ of students only preferred the visual, aural, reading/writing and kinesthetic modes, respectively. A further analysis showed that $72.6 \%$ of students preferred multiple learning styles.

Conclusions: The results of this study can provide useful information for improving the quality of the teaching and learning experiences of students. However, more research on this topic needs to be undertaken before the association between learning style preferences and teaching and learning strategies is more clearly understood.

Keywords: Learning style, learning preference, the VARK, medical students

\section{Introduction}

Although there are a large number of learning styles, strategies and approaches based on different psychological constructs $^{1,2}$ there is a great deal of interest amongst educators in identifying whether learners are predominantly visual, auditory, reading/writing or kinesthetic learners. ${ }^{3}$ Students' approaches to learning can be influenced by the perception that they gain from their learning environment.

In preclinical years, medical students experience different learning environments whilst acquiring large amounts of information. To achieve this, each student must adapt his or her own learning style preference. Keefe stated that "Learning style is the composite of cognitive, affective and physiological characteristics that serve as relatively stable indicators of how a learner perceives, interacts and responds to the learning environment". ${ }^{4}$

He also suggested that teachers need to understand the learning strategies of students in order to facilitate their learning. It has also been argued that medical teachers should not only possess content knowledge but should also be acquainted with their learner's attributes. ${ }^{5}$

Many factors can influence student learning styles. These factors are: gender, age, academic achievement, brain processing, culture and creative thinking. ${ }^{6}$ What is more, students use different sensory modalities for assimilating knowledge and information. ${ }^{7}$ Four sensory modalities described by Fleming are: visual (V), Aural (A), Reading/Writing (R) and Kinesthetic (K), which is called as VARK. ${ }^{8}$ Briefly, a visual student prefers to learn visually and an aural student uses listening techniques to learn. Some students use reading and writing as their first preferences for assimilating and accommodating to information. A kinesthetic student experiences learning by all sensory channels, including somatosensory, auditory, olfactory, gustative and visual. ${ }^{9}$ The Visual-Aural-Read/writeKinesthetic (VARK) questionnaire has been specifically developed in the context of modalities and strategies of 
learning styles. Over the past year, the VARK has been used in some countries to assess learning style preferences of students. In reviewing the literature on the VARK, for instance, cross sectional studies show that students preferred a multimodal learning style. ${ }^{10-12}$ Although there are inconsistent results in some studies little is known about the distribution of learning preferences of medical students in Saudi Arabia medical education where the culture and medical education differ greatly from Western countries.

Therefore, the purpose of the study was to categorize learning preferences of medical students using the latest English version 7.0 of the VARK questionnaire. One question guided the study: What is the distribution of the learning style preferences of medical students at a single institute in Saudi Arabia.

\section{Methods}

\section{Setting and participants}

A descriptive cross sectional study was conducted in 2011. Of the 210 third-and fourth-year medical students at King Saud Bin Abdul Aziz University for Health Sciences, King Fahad Medical College, 146 volunteers (74 male and 72 female) participated in this study.

\section{Instrument}

Version 7 of the VARK questionnaire was used in this study. The questionnaire measures four perceptual preferences (V, A, R and K). It consists of 16 questions with four options each. The purpose of each question is to categorize the learning style preferences of respondents. Respondents can choose more than one option for identifying the preferences for multiple learning styles. Satisfactory levels of reliability and validity of the VARK have been reported using factor analysis techniques. ${ }^{13}$

\section{Procedures}

In 2011, we distributed the VARK questionnaire to thirdand fourth-year medical students during regular classes and then asked students to complete the questionnaire and return it to the investigators. We explained that the VARK questionnaire was designed to measure the distribution of learning styles preferences of students and that we would use the study findings for research purposes. The study was approved by the Institutional Review Board of King Fahad Medical, King Saud Bin Abdul Aziz University for Health Sciences, Riyadh, Saudi Arabia.

\section{Statistical analyses}

The distributions of the VARK preferences were calculated in accordance with the guidelines given in the VARK website. ${ }^{8}$ Descriptive statistics were used for each VARK component. To calculate the percentage of students for each VARK component the number of students who preferred each learning style modality was divided by the total number of students $(n=146)$.

\section{Results}

In our study, the response rate was 70\%. Mean and standard deviation for each VARK component are presented in Table 1.

Table 1. Mean and standard deviation for the VARK questionnaire administered to 146 medical students at King Saud Bin Abdul Aziz University for Health Sciences, King Fahad Medical College, Saudi Arabia, 2011

\begin{tabular}{lcc}
\hline VARK & Mean & SD \\
\hline Visual & 5.3 & 2.9 \\
Auditory & 6.6 & 3.2 \\
Reading/Writing & 4.7 & 2.6 \\
Kinesthetic & 6.4 & 3.1 \\
\hline
\end{tabular}

Figure 1 illustrates that $5.5 \%, 11.6 \%, 2.1 \%$, and $8.2 \%$ of students only preferred the visual, aural, reading/writing and kinesthetic modes, respectively. This simply indicates that only $27.4 \%$ of students preferred to learn by a single sensory modality (visual, auditory, reading/writing, or kinesthetic).

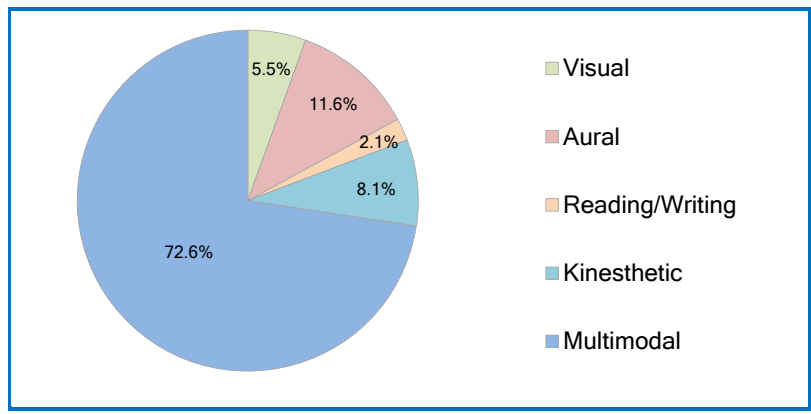

Figure 1. The percentages of students with singular and multimodal learning preferences $(n=146)$

However, a further analysis showed that the vast majority of students $(72.6 \%)$ preferred to learn by multiple sensory modalities. Of these, $34.9 \%, 42.5 \%$ and $22.6 \%$ were bimodal, trimodal and quadmodal, respectively (Figure 2).

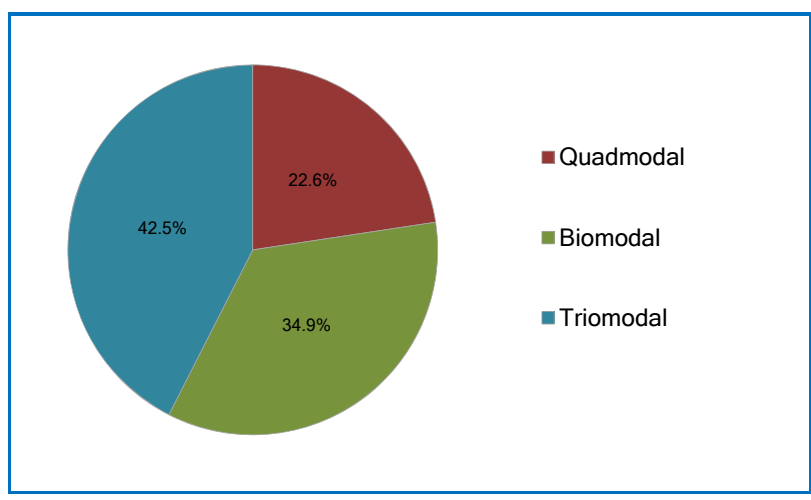

Figure 2. The percentages of students who preferred two, three or four modes of the learning style preferences $(n=146)$

From Figure 3 we can see that the dominant learning preferences of the trimodal students were visual, auditory and kinesthetic (17.9\%). Only $5.7 \%$ of students preferred visual, reading/writing and kinesthetic. Figure 3 also shows that the dominant learning performances of the bimodal 
students were auditory and kinesthetic (12.3\%). This figure also shows that $22.6 \%$ of students who preferred quadmodal use all components for learning, i.e. visual, auditory, reading/writing, and kinesthetic.

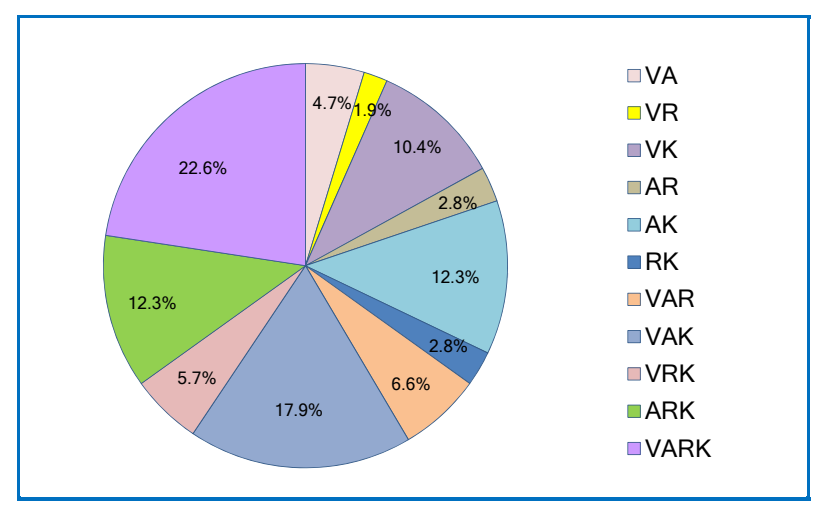

Figure 3. The percentages of students with a preferred combination of learning styles $(n=146)$

\section{Discussion}

This study was carried out to gain an understanding of the learning preferences of Saudi Arabian medical students and to answer the question: What is the distribution of the learning style preferences of medical students? In general, the findings of this study provide insight into the ways that our medical students learn in relation to the subject of study. We think our study may help to shed light on medical students learning development.

We found that many students preferred to learn by more than one mode of information presentation. The VARK questionnaire is widely used by researchers to identify the learning preference of students., 12, 14, 15 Learning style varies from one group to another based on culture, the nature of the studies and the characteristics of students. In our study the Mean VARK scores for aural (6.6) and kinesthetic learners (6.4) were more than that for visual (5.3) and reading /writing learners (4.7). This could be due to the fact that Saudi Arabian students are heavily involved in new technology. A study in Malaysia showed that the mean VARK scores of kinesthetic (5.0) and read/write students (4.9) were more than auditory (4.7) and visual learners (3.1). ${ }^{14}$ It has been also reported that science and engineering students were kinesthetic learners whereas business students were reading/writing learners. ${ }^{16}$

Our findings regarding multimodal preferences in Saudi Arabian students are in agreement with the study that has been reported for American medical students. ${ }^{12}$ Solvie and Kloek argued "students' perceptions of their learning experience and understanding of course content were considered in analysing the effectiveness of technology used to address multiple learning styles in a constructivist environment". ${ }^{17}$ Further work is required to establish this. Medical teachers can develop their pedagogical strategies if they know the preferred modes of learning of their students. ${ }^{9}$ This allows them to shift from their own preferred mode of teaching towards the learning preferences of medical students which may help to develop their knowledge, skills and attitudes and that may enable them to become a competent student. ${ }^{18-21}$

Visual learners should be stimulated with depictions of information in charts, graphs, flow charts, and all the symbolic arrows, circles and other devices that instructors use to represent what could have been presented in words.12 Auditory learning is achieved through listening during peer instruction ${ }^{22,23}$ collaborative testing ${ }^{24,25}$, debate $^{26}$, games ${ }^{27}$, and answering questions.28 Manipulating models $^{29}$ and role playing ${ }^{30}$ satisfies kinesthetic and tactile learners. Reading /writing learners can be approached with information depicted in words. Few studies have reported an increase in student achievement by using simulation and games. ${ }^{20}$ To achieve effective learning it has been reported that students must read, write and talk about their tasks, relate them to prior experience and knowledge and engage in application. ${ }^{20}$

\section{Limitations of the study}

This study has several limitations. First, a convenience sample of students from a single institute was used. Therefore the sample may have been biased and might not represent the population of medical students across Saudi Arabia. Further studies using multiple centers with a large sample size on the current topic are therefore recommended. Second, as there is a large number of learning styles based on different psychological constructs and assessed using a range of measurement instruments, it is impossible to categorize students into fixed learning styles as many other factors can influence the learning style preferences of the students. However, understanding how the natural features of students affect the way they process information is important for medical educators.

\section{Conclusion}

The results of this study can provide useful information for improving the quality of the teaching and learning experiences of students. Therefore it is important that medical educators need to be aware of classes containing students where specific learning styles vary. However, more research on this topic needs to be undertaken before the association between learning style preferences and teaching and learning strategies is more clearly understood.

\section{Conflict of Interest}

The authors declare that they have no conflict of interest.

\section{References}

1. Kolb D. Learning style inventory (revised edition) Boston: McBer; 1985.

2. Biggs J. From theory to practice: a cognitive systems approach Higher education Research and Development.12:73-86;1992. 
3. Dryden G, Vos J. The learning revolution: to change the way the world learns. Stafford: Neteork Educational Press Ltd; 2001.

4. Keefe J. Learning style: theory and practice. Reston: National Association of Secondary School Principals; 1987.

5. Gudmundsdottir SS. Pedagogical content knowledge in social studies. Scand J Educ Res. 1987;31( 2):59-70.

6. Honigsfeld A. A comparative analysis of the learning styles of adolescents from diverse nations by age, gender, academic achievement level and nationality The Humanities and Social Sciences 2001;62:969.

7. Slater J, Lujan H, DiCarlo S. Does gender influnce learning style preference of first-year medical students. Adv Physiol Educ. 2007;31:336-42.

8. Fleming N. VARK: a guide to learning styles 2007 [cited 201124 July 2011]; Available from: http://www.varklearn.com/english/index.asp.

9. Fleming N. I'm different; not dumb: modes of presentation (VARK) in the tertiary classroom. In: Canberra Z, editor. Research and Developlement in Higher Education: Proceedings of the 1995 Annual Conference of the Higher Education and Research Development Society of Australia. Australia1995. p. 303-18.

10. Baykan Z, Naçar M. Learning styles of first-year medical students attending Erciyes University in Kayseri, Turkey. Adv Physiol Educ. 2007;31(2):158-60.

11. Alkhasawneh I, Mrayyan M, Docherty C, Alashram S, Yousef H. Problem-based learning (PBL): assessing students' learning preferences using VARK. Nurse Educ Today. 2008;28(5):572-9.

12. Lujan H, DiCarlo S. First-year medical students prefer multiple learning styles. Adv Physiol Educ. 2006;30(1):13-6.

13. Leite W, Svinicki M, Shi Y. Attempted validation of the scores of the VARK: learning styles inventory with multitrait-multimethod confirmatory factor analysis models Educational and Psychological Measurement. 2010; 70(2):323-39.

14. Kumar L, Voralu K, Pani S, Sethuraman K. Predominant learning styles adopted by AIMST university students in Malaysia. South East Asean Journal of Medical Education. 2009;3:37-46.

15. Murphy R, Gray S, Straja S, Bogert M. Students learning preferences and teaching implications. J Dent Educ. 2004;68(8):859-66.

16. Tierney J, Brunton E. Learning styles: a factor in course choice. B.Sc. Project in Health \& Leisure, Institute of Technology. Tralee2005; Available from:

https://www.ittralee.ie/en/InformationFor/Staff/Teachingan dLearningUnit/Journals2006-2007/Title,15322,en.html.

17. Solvie P, Kloek M. Using technology tools to engage students with multiple learning styles in a constructivist learning environment. Contemporary Issues in Technology and Teacher Education. 2007;7(2):7-27.

18. Collins J. Education techniques for lifelong learning: principles of adult learning. Radiographics. 2004;24(5):1483-9.

19. Forrest S. Learning and teaching: the reciprocal link. J Contain Educ Nurs. 2004;35(2):74-79.

20. Lujan H, DiCarlo S. Too much teaching, not enough learning: what is the solution? . Adv Physiol Educ. 2006;30(1):17-22.

21. Miller J. Enhancement of achievement and attitudes through individualized learning-style presentations of two allied health courses. J Allied Health. 1998;27(3):150-6.

22. Cortright R, Collins H, DiCarlo S. Peer instruction enhanced meaningful learning: ability to solve novel problems. Adv Physiol Educ. 2005;29(2):107-11.

23. Rao S, DiCarlo S. Peer instruction improves performance on quizzes. Adv Physiol Educ. 2000;24(1):51-5.

24. Cortright R, Collins H, Rodenbaugh D, DiCarlo S. Student retention of course content is improved by collaborative-group testing. Adv Physiol Educ. 2003;27(1-4):102-8.

25. Rao S, Collins H, DiCarlo S. Collaborative testing enhances student learning. Adv Physiol Educ. 2002;26(14):37-41.

26. Scannapieco F. Formal debate: an active learning strategy. J Dent Educ. 1997;61(12):955-61.

27. GGreeblaw P, Wyman F. The teaching effectiveness of games in collegiate business courses.

Simulations Games 1985;16:251-8. Simulation \& Gaming. 1973;4( ):259-94.

28. DiCarlo S, Collins H. Colored letters: a tool to increase class participation in a large classroom. Adv Physiol Educ. 2001;25:143.

29. Chan V, Pisegna J, Rosian R, DiCarlo S. Construction of a model demonstrating neural pathways and reflex arcs. Adv Physiol Educ. 1996;271(6 pt 3):S14-42.

30. Kuipers J, Clemens D. Do I dare? Using role-play as a teaching strategy. J Psychosoc Nurs Ment Health Serv. 1998;36(7):12-7. 